\title{
Prevalence of and related risk factors in oral mucosa diseases among residents in the Baoshan District of Shanghai, China
}

\author{
Shuyun Ge ${ }^{1}$, Lin Liu ${ }^{2,3}$, Qi Zhou ${ }^{4}$, Binbin Lou ${ }^{5}$, Zengtong Zhou ${ }^{1}$, Jianing Lou ${ }^{\text {Corresp., } 5}$, Yuan Fan ${ }^{\text {Corresp. } 2,3}$ \\ ${ }^{1}$ Department of Oral Medicine, Shanghai Key Laboratory of Stomatology, Shanghai Ninth People's Hospital, Shanghai Jiao Tong University School of \\ Medicine, Shanghai, China \\ 2 Department of Oral Medicine, Affiliated Hospital of Stomatology, Nanjing Medical University, Nanjing, China \\ 3 Jiangsu Key Laboratory of Oral Diseases, Nanjing Medical University, Nanjing, China \\ 4 Medical Association Office of Shanghai Baoshan District, Shanghai, China \\ 5 Department of Stomatology, Shanghai General Hospital of Nanjing Medical University, Shanghai, China \\ Corresponding Authors: Jianing Lou, Yuan Fan \\ Email address: 18939829711@163.com, fanyuan@njmu.edu.cn
}

Background: Oral mucosal diseases (OMDs) encompass a variety of different types of diseases. Our aim was to evaluate the prevalence and related risk factors of OMDs among residents in the Baoshan District of Shanghai, China, and provide a scientific basis for prevention and control strategies. Methods: A sample of 653 residents aged 17 to 92 years from the Baoshan community was investigated in 2014. Each resident was surveyed by questionnaire to evaluate their oral mucosa and oral mucosa examinations were conducted. We followed up with 607 residents in 2018. All data were statistically analyzed using the SPSS 25.0 software package (Chicago, IL, USA) at the general population, gender and age levels. $A X^{2}$ test was used to compare rates of risk factors and logistic regression analysis was used to detect the correlation between disease and risk factors. Results: The prevalence rate of OMDs was found to be $9.19 \%-9.56 \%$ (2014-2018). The most common OMDs were atrophic glossitis (1.84\%), recurrent aphthous ulcer (RAU, 1.68\%), burning mouth syndrome (BMS, 1.38\%), oral lichen planus (OLP, 1.23\%) and traumatic ulcers (1.23\%). The prevalence of RAU and BMS in different age groups was significantly different. Tobacco and alcohol use and psychological factors in the OMDs group were higher than the no-OMDs group. Systemic diseases including diabetes mellitus (DM) was significantly relevant to OLP. Conclusion: Age, tobacco and alcohol use, and psychological factor correlated strongly with the occurrence and development of OMDs, and they should be the focus of primary prevention. General epidemiological studies suggested that OLP was closely related to DM. 


\section{Prevalence of and related risk factors in oral mucosa}

2 diseases among residents in the Baoshan District of

\section{Shanghai, China}

4

5

6 7
Shuyun $\mathrm{Ge}^{1}$, Lin $\mathrm{Liu}^{2,3}$, Qi Zhou ${ }^{4}$, Binbin Lou ${ }^{5}$, Zengtong Zhou ${ }^{1}$, Jianing Lou ${ }^{5 *}$, Yuan Fan²,3*

${ }^{1}$ Department of Oral Medicine, Shanghai Key Laboratory of Stomatology, Shanghai Ninth People's Hospital, Shanghai Jiao Tong University School of Medicine, Shanghai 200011, P. R.

China

2 Jiangsu Key Laboratory of Oral Diseases, Nanjing Medical University, Nanjing, P. R. China

${ }^{3}$ Department of Oral Medicine, Affiliated Hospital of Stomatology, Nanjing Medical University, Nanjing, P. R. China

${ }^{4}$ Medical Association Office of Shanghai Baoshan District; Shanghai 201900, P. R. China

${ }^{5}$ Department of Stomatology, Shanghai General Hospital of Nanjing Medical University, Shanghai 201620, P. R. China

Corresponding Author:

${ }^{1}$ Jianing Lou

Department of Stomatology, Shanghai General Hospital of Nanjing Medical University, Shanghai 201620, P. R. China

Email address: 18939829711@163.com

${ }^{2}$ Yuan Fan

Jiangsu Key Laboratory of Oral Diseases, Nanjing Medical University, Nanjing, China

Email address: fanyuan@njmu.edu.cn 


\section{Abstract}

33 Background: Oral mucosal diseases (OMDs) encompass a variety of different types of

34 diseases. Our aim was to evaluate the prevalence and related risk factors of OMDs among

35 residents in the Baoshan District of Shanghai, China, and provide a scientific basis for prevention

36 and control strategies.

37 Methods: A sample of 653 residents aged 17 to 92 years from the Baoshan community was investigated in 2014. Each resident was surveyed by questionnaire to evaluate their oral mucosa and oral mucosa examinations were conducted. We followed up with 607 residents in 2018. All data were statistically analyzed using the SPSS 25.0 software package (Chicago, IL, USA) at the general population, gender and age levels. A $\mathrm{X}^{2}$ test was used to compare rates of risk factors and logistic regression analysis was used to detect the correlation between disease and risk 43 factors.

Results: The prevalence rate of OMDs was found to be $9.19 \%-9.56 \%$ (2014-2018). The most common OMDs were atrophic glossitis (1.84\%), recurrent aphthous ulcer (RAU, 1.68\%), burning mouth syndrome (BMS, 1.38\%), oral lichen planus (OLP, 1.23\%) and traumatic ulcers $(1.23 \%)$. The prevalence of RAU and BMS in different age groups was significantly different. Tobacco and alcohol use and psychological factors in the OMDs group were higher than the noOMDs group. Systemic diseases including diabetes mellitus (DM) was significantly relevant to OLP.

51 Conclusion: Age, tobacco and alcohol use, and psychological factor correlated strongly with the occurrence and development of OMDs, and they should be the focus of primary prevention. General epidemiological studies suggested that OLP was closely related to DM.

Keywords: Diabetes mellitus, epidemiology investigation, oral lichen planus, oral mucosal diseases, prevalence rate, risk factors 
59 Introduction:

60 Oral mucosal diseases (OMDs) occur in the oral mucosa and most of them are associated with 61 systemic diseases (Porter, Mercadante \& Fedele, 2017). The various kinds of OMDs include 62 recurrent aphthous ulcer (RAU), burning mouth syndrome (BMS), oral lichen planus (OLP), oral

63 leukoplakia (OLK), oral erythroplakia (OE), traumatic ulcers, and so on. Except for traumatic

64 ulcers, the etiology of most OMDs is unknown. Some of these diseases have potential malignancies that seriously affect quality of life and are even fatal. Oral Potentially Malignant Disorders (OPMDs) refers to diseases that may cause oral cancer (Wang et al., 2014; Dionne et al., 2015). OLP is a common OPMDs, with a prevalence rate of $0.1 \%-4.0 \%$ (Kurago, 2016) and a cancerization rate of 0\%-12\% (van der Meij, Schepman \& van der Waal, 2003). OLK is another common OPMDs, with a cancerization rate of $10-30 \%$ and an average time to cancer onset of 4.0-8.1 years (Petti, 2003; Warnakulasuriya et al., 2011). OE is the most cancer-prone OPMDs, with a cancerization rate as high as 50\% (Villa, Villa \& Abati, 2011). mechanism are unclear. Generally, there are no specific treatments and no effective chemical prophylaxis drugs for cancer (Ribeiro et al., 2010; van Monsjou et al., 2013). OPMDs have a poor prognosis and the overall cancerization rate is close to $4.32 \%$ (Wang et al., 2014). Due to the prolonged course of the disorder, and the risk of cancer, patients often suffer physical and mental pain. Early diagnosis of OPMDs is of great significance for the prevention of oral malignant tumors (McCullough, Prasad \& Farah, 2010; Amagasa, Yamashiro \& Uzawa, 2011). Shanghai, with a population of more than 24 million, was chosen as the location of the study because work stress, mental stress, environmental changes, dietary changes and accelerated life rhythms are thought to increase the prevalence of OMDs. Therefore, it is of great interest to understand the epidemiological characteristics of OMDs in this city and analyze the risk factors associated with those diseases. Early prevention and treatment of OMDs and early detection of

84 high-risk groups of oral cancer will improve people's quality of life. 
85

86

87

88

89

90

91

92

93

94

95

96

97

The prevalence of OMDs was studied in a cross-sectional study using general epidemiology. Our subjects were either selected from the general population in the Shanghai region or were oral outpatients. There is currently little available data on the OMDs in the Shanghai population. In the last 30 years, epidemiological investigations of OMDs have been mostly based on analysis of clinical data, or case-controlled studies (Ikeda et al., 1995; Mumcu et al., 2005; Splieth et al., 2007; Pentenero et al., 2008; Mansour Ghanaei et al., 2013; Do et al., 2014; ).

Little work has been done in the epidemiology of OMDs, especially descriptive epidemiology; most previous studies are investigations of specific age groups or special diseases (Xu et al., 1981; Qi, 2008). In order to make an accurate diagnosis of an OMD, good theoretical knowledge and sufficient clinical experience was required, especially considering the low prevalence of OMDs in the general population and large sample size in the survey.

In this article, we report on the epidemiological characteristics of OMDs in the Baoshan District of Shanghai, analyze the risk factors of the diseases to better understand prevalence and epidemic characteristics, and establish a scientific basis for the prevention and treatment of OMDs.

\section{Materials and methods:}

\section{Research objectives}

We used multistage stratified random sampling and field surveys to investigate the population of the Baoshan District of Shanghai in 2014. Four neighborhood committees were selected by a random cluster method, and entire families were selected for oral examination according to house number. We calculated maximum sample size using the OMD prevalence rate of $14.93 \%$ (range 14.93-29.3\% according to Xu et al., 1981; Cao et al., 1988). The formula $[\mathrm{n}=\mathrm{t} 2 * \mathrm{p} / \mathrm{d}$ (1 - P) $2, \mathrm{p}=14.93 \%, \mathrm{~d}=0.1 * \mathrm{p}$, take $\boldsymbol{\alpha}=0.05, \mathrm{t}=1.96]$ yielded a theoretical figure of 2189 people. Unfortunately, this study was only able to sample 653 people. We conducted follow up with patients 4 years later to assess the long-term effects of persistent risk factors on the course 
112 of the disease. The procedures were approved by the Ethical Committee of the Stomatological

113 Hospital Affiliated to Nanjing Medical University (PJ2014-132) and the ethical committee of

114 Shanghai First People's Hospital (2019KY063).

115

116 Questionnaire design and oral mucosa examination

117 The survey was prepared according to the World Health Organization's (2013, WHO) oral health

118 assessment form guidelines. It was designed to evaluate the condition of the oral mucosa. The

119 questionnaire includes demographics (name, gender, age, nationality, place of birth, length of

120 residence in Shanghai, education level, marital status, occupation, etc.), smoking habits (current,

121 former or never), drinking habits (current, former or never), systemic disease (diabetes,

122 hypertension, coronary heart disease and cerebrovascular diseases), and mental status (stress,

123 anxiety). For the purposes of the survey, we considered systemic disease to be identified if the

124 resident was diagnosed by a doctor. Gestational diabetes was not included in this survey.

125 Oral mucosa examination followed the clinical diagnostic criteria proposed by the WHO

126 (Kramer et al., 1980). Portable halogen lamps, disposable retractors, and mouth mirrors were

127 used in this study. When performing oral mucosa examinations, the intraoral and perioral

128 mucosa and soft tissue were comprehensively examined for each subject in the following

129 systematic order: lip, corner of mouth, cheek, tongue, bottom of mouth, hard and soft palate,

130 alveolar ridge and gingiva. When residents were clinically diagnosed with OPMDs, including

131 OLP or OLK, we referred them to our hospital for laboratory tests and biopsies. Expert

132 pathologists confirmed the final diagnosis. Informed consent was obtained prior to the initiation

133 of the examination. Inspectors were trained to ensure that guidelines for observation were

134 interpreted uniformly and that mucosal diseases were documented correctly. Calibration

135 exercises were repeated every month. Ten inspectors participated in the study, six of those

136 participated throughout the whole study.

137

138 Statistical methods:

Peer) reviewing PDF | (2019:08:40507:2:0:NEW 13 Jan 2020) 
139 All data were analyzed statistically using the SPSS 25.0 software package (Chicago, IL, USA).

140 Two people were responsible for data entry and analysis. A $\mathrm{X}^{2}$ test was used to compare the rates

141 of risk factors. Logistic regression analysis was used to detect correlation between disease and

142 risk factors. Results were considered significant if $\mathrm{p}<0.05$.

144 Results:

145 Survey results

146 A total of 653 permanent residents were surveyed in 2014, including 337 males and 316 females.

147 We followed up with approximately 93\% of those residents in the 2018 survey: 607 residents

148 (311 men and 296 women). The remaining 7\% were lost to follow up.

149 The education levels of the test subjects were mainly middle school (and below) level,

150 mostly retired people. The average age of the respondents in 2014 was 66.05 years old (17-92),

151 and the average age in 2018 was 67.38 years old (18-91).

152

153

\section{Prevalence of OMDs}

154 In the 2014 survey, a total of 60 people (9.19\%) had OMDs, which included 12 cases of atrophic

155 glossitis (1.84\%), 11 cases of RAU (1.68\%), nine cases of burning mouth syndrome (BMS)

$156(1.38 \%)$, eight cases of OLP (1.23\%), eight cases of traumatic ulcer $(1.23 \%)$, six cases of

157 furrowed tongue $(0.92 \%)$, three cases of geographic tongue $(0.46 \%)$, one case of cheilitis

$158(0.15 \%)$, one case of angular cheilitis (0.15\%) and one case of leukoplakia $(0.15 \%)$. The results

159 showed that the OMDs were mostly localized at the back of tongue, buccal mucosa, labial

160 mucosa and gingival (gums).

161

In 2018, 58 people (9.56\%) had OMDs (46 people were lost to follow-up, which included

16241 with no OMDs and five with OMDs). Compared to 2014, there were ten cases of decreased

163 OMDs and nine cases of new OMDs as follows: 11 cases of atrophic glossitis $(1.81 \%$, one case

164 lost, no new), nine cases of RAU (1.48\%, two cases lost, no new), seven cases with furrowed

165 tongue $(1.15 \%$, none lost, one new), nine cases of OLP (1.48\%, none lost, one new case of DM), 
166

167

168

169

170

171

172

173

174

175

176

177

178

179

180

181

182

183

184

185

186

187

188

189

190

191

192

seven cases of traumatic ulcer $(1.15 \%$, none lost, eight cases of decrease, seven case new), one case of geographic tongue $(0.16 \%$, one lost, one case of decrease). One case each of cheilitis, angular cheilitis and leukoplakia ( $0.16 \%$, none lost, no increase, no decrease).

\section{Distribution of diseases in males and females and in different age groups in 2014}

The prevalence rate in females was higher than in males in cases of RAU, BMS and OLP $(\mathrm{p}<0.05$; Figure 1). In addition, the overall prevalence of OMDs in females was also higher than in men $(\mathrm{p}<0.05)$. The cases of RAU and BMS were statistically significant in different age groups. The prevalence of RAU in 15-29 year-olds and 30-39 year-olds was significantly higher than that in the older age groups $(50-59,60-69,70-79)(\mathrm{p}<0.01)$, but there was no significant difference between the two groups ( $\mathrm{p}>0.05$ ). We found BMS more frequently in people over 40 years old, prevalence increased with age, and was significantly higher in the 70-79 age group than in the 40-49 group ( $<<0.01)$. OLP was detected in the population over 40 years old, and there was no significant difference among age groups (Figure 2).

\section{Effect of living habits and mental state on OMDs in 2014}

Respondents generally had a light diet. There were 107 (16.39\%) smokers (people who had smoked for over three years) and 93 (14.24\%) who were drinkers (millet wine and beer). The rate of smoking $(\mathrm{p}=0.01)$ and alcohol consumption $(\mathrm{p}=0.00003)$ in persons with OMDs was higher compared to those with no OMDs (Figure 3). Our survey showed that 87 people were had mental anxiety and 24 people felt higher than normal stress in life. Those with OMDs were had a higher rate of anxiety compared to those with no OMDs $(p=0.0002)$. However, there was no statistically significant difference in stress between persons with OMDs and no OMDs ( $\mathrm{p}=0$. 903) (Figure 4). The sample group included 97.5\% people in a stable family relationship, $28.1 \%$ with no friends and $36.8 \%$ having a little interpersonal communication. The vast majority $(96.2 \%)$ of respondents had no adverse life experiences in past 12 months.

Peer] reviewing PDF | (2019:08:40507:2:0:NEW 13 Jan 2020) 
193

194

195

196

197

198

199

200

201

202

203

204

205

206

207

208

209

210

211

212

213

214

215

216

\section{Discussion:}

218

219

\section{Discussion:}

\section{Analysis of risk factors related to OMDs and OLP in 2014}

This study found that there was no significant difference in the prevalence of OMDs between men and women in the population of Baoshan, Shanghai. It also found that the occurrence and prevalence of OMDs, particularly atrophic glossitis, RAU, BMS and OLP, were positively correlated with increasing age among residents.

The study also found that rates of smoking, drinking and mental anxiety in the OMD population were significantly higher than in the healthy population, but that there was no statistical difference. Systemic diseases, such as cardiovascular and cerebrovascular hypertension $(p=0.025)$ and metabolic diseases or diabetes $(p=0.028)$, were closely related to OMDs. Systemic diseases such as cardiovascular and cerebrovascular hypertension $(\mathrm{p}=0.025)$, and metabolic diseases diabetes $(\mathrm{p}=0.028)$ were closely related to OMDs. The investigation group theorized that the high number of elderly patients would have proportionally higher incidence of dental cavities and removable dentures and presumed that incidence of traumatic ulcers would also increase proportionally (Table 1).

OLP was more common in the elderly age group (40-60 years) and occurred in more females than males. In this study, OLPs mostly occurred in the buccal region. The cause of OLP is complex and still unknown. The WHO defines OLP as a potentially malignant disease of the oral mucosa. Currently, there is no specific treatment for OLP in clinical practice. As a result, the course of the disease is prolonged and the condition often recurs. OLP may develop into cancer, which has a vital impact on patients' physical and mental health. The results of the general epidemiological investigation showed that there were eight community residents $(1.23 \%)$ with OLP: two males and six females (male to female ratio 1:3). OLP was significantly associated with age, smoking and diabetes mellitus $(\mathrm{p}<0.01)$ (Table 2$)$.

In this survey, we found that the prevalence rate of OMDs in the Shanghai region varied from 9.19\% (2014) to $9.56 \%$ (2018). The most prevalent OMDs were incidence atrophic glossitis, 
220 RAU, BMS and OLP. Other surveys in Shanghai have found higher OMD prevalence rates: $\mathrm{Xu}$ 221 et al. (1981) found a prevalence rate in 1978 of 14.93\%; Cao et al. (1988) found a prevalence 222 rate in the over 60s age group of $29.3 \%$; and a large-scale epidemiological investigation 223 conducted by Feng et al. (2015) among 11,054 people in 2012 found a prevalence rate of 10.8\%. 224 Work, psychological stress, environmental changes, changes in diet structure and the 225 accelerated pace of life are all thought to lead to an increased prevalence of the OMD condition. In our study, OMDs were positively correlated with age, which supports the findings of Cao et al. (1988). Therefore, preventive measures should be commensurate with the risk factor of increasing age. Atrophic glossitis occurred mainly in the elderly over 60 years of age in our study, which was consistent with its etiology: chronic anemia, lack of nicotinic acid, Sjogren's syndrome and Candida infection. Among people aged 30-39 years, there has been a significant increase in the prevalence of RAU which occurs in more female patients than male patients. were ill.

In 2014, eight residents with traumatic ulcers were recommended treatment at the time of the first examination. During the 2018 follow-up, all eight patients dealt with trauma factors and their ulcers had disappeared. It indicated that community epidemiological surveys have, in addition to a diagnostic function, a preventative function that may contribute to the early treatment of residents, reduce cancer risk, and limit the number of untreated ulcers. The followup indicated that early prevention and control of mucosal diseases was of great significance. Seven new cases of traumatic ulcers found in the current investigation were treated and the patients were informed of the risks. Many of the interviewees were elderly people with high rates of dental cavities and periodontitis. The elderly lacked the awareness to treat cavities and

243 maintain dentures. As a result of the study, detection rates of traumatic ulcers increased, in 244 particular, those occurring at the denture base covering the gums and palate areas. Glossy, traumatic ulcers were associated with residual roots and crowns. With continuous improvement 
246 in living standards, the rate of visits to medical centers to treat traumatic ulcers has gradually

247 increased.

248 Our study showed that the prevalence of OMDs in patients who smoke, drink and

249 experience anxiety was higher than in those who were healthy. Many studies have confirmed that

250 tobacco and alcohol are closely related to OMDs and are risk factors for OMDs (Shulman, Beach

251 \& Rivera-Hidalgo, 2004; Dundar \& Ilhan Kal, 2007; Pentenero et al., 2008; Mohamed \&

252 Janakiram, 2014). In past surveys, the prevalence of BMS was extremely low and rarely

253 detected. However, the present survey found that the prevalence of BMS increased in middle-

254 aged and older women in the perimenopausal and postmenopausal stages, and was accompanied

255 by obvious changes in psychological and mental state. This might be due to the gradual

256 acceleration of the pace of life in recent years, and subsequent increase in psychological

257 problems. The causes of many diseases, especially chronic diseases, are no longer a simple

258 biological factor, but also include many social, environmental and psychological factors.

259 Alcohol, tobacco, stress and mental factors associated with the occurrence and development of

260 OMDs should be the focus of primary prevention (O'Sullivan, 2011; Mendes et al., 2012).

261 It is noteworthy that the 4-year follow-up of community residents with OMDs suggested

262 that systemic metabolic diseases, such as DM, were closely related to OPMDs, such as OLP.

263 This result is consistent with the results of a large-scale oral health epidemiological investigation,

264 in which we participated, in 2012 in Shanghai (Feng et al., 2015). The OMD group is comprised

265 of many oral diseases and their prevention and control is difficult. At present, prevention and

266 treatment of OMDs are still the main factors in the three-levels of disease control.

267

268 Conclusion:

269 Early prevention and control of mucosal disease is of great importance. It is necessary to

270 better understand the prevalence of OMDs among Shanghai residents, and to carry out effective

271 intervention activities through better health education, policy formulation and the creation of a

272 supportive environment. Further, there is a need to reduce related risk factors, to promote the 
273 health of oral mucosa, and to improve the quality of life of the population. Finally, we consider it 274 imperative to elevate the importance of primary prevention and treatment of OMDs.

275

276 Funding:

277 1. Science and technology development fund project of Baoshan, Shanghai (12E62)

278 2. The national natural science foundation of China- youth project (30700944)

279 3. The science research project of Shanghai health bureau (2012092)

280 4. The National clinical key specialty construction project (2013-544)

281 5. The National Institutes of Health (USA) Research Grant (R01 DK110273-01A1)

282 6. National Natural Science Foundation of China (81970941)

283 7. A Project Funded by the Priority Academic Program Development of Jiangsu Higher

284 Education Institutions (PAPD 2018-87)

286 Acknowledgements:

287 We thank all the physicians from the Department of Oral Mucosal Diseases, Ninth People's 288 Hospital, Shanghai Jiao Tong University School of Medicine and Friendship Street Community 289 Health Service Center of Shanghai Baoshan District for Participating in our epidemiological 290 survey and performing the oral mucosal examination.

292 Disclosure:

293 The author reports no conflicts of interest in this work. 
297 Amagasa T, Yamashiro M, Uzawa N. 2011. Oral premalignant lesions: from a clinical perspective. Int J Clin Oncol 16:5-14. DOI: 10.1007/s10147-010-0157-3.

299

300

301

302

303

304

305

306

307

308

309

310

311

312

313

314

315

316

317

318

319

320

321

322

Cao HK, Le FY, Chen JH, Lu H, Shi HB, Shen ZY, Hu HF, Jin WC, Hu LY, Tang GY, Chen YP. 1988. Epidemiological study on oral mucosal diseases of 3,091 elderly people in Shanghai. Oral Med 8:169-171.

Dionne KR, Warnakulasuriya S, Zain RB, Cheong SC. 2015. Potentially malignant disorders of the oral cavity: current practice and future directions in the clinic and laboratory. Int $J$ Cancer 136:503-515. DOI: 10.1002/ijc.28754.

Do LG, Spencer AJ, Dost F, Farah CS. 2014. Oral mucosal lesions: findings from the Australian National Survey of Adult Oral Health. Aust Dent J 59:114-120. DOI: 10.1111/adj.12143.

Dundar N, Ilhan Kal B. 2007. Oral mucosal conditions and risk factors among elderly in a Turkish school of dentistry. Gerontology 53:165-172. DOI: 10.1159/000098415.

Feng J, Zhou Z, Shen X, Wang Y, Shi L, Hu Y, Sun H, Liu W. 2015. Prevalence and distribution of oral mucosal lesions: a cross-sectional study in Shanghai, China. J Oral Pathol Med 44:490-494. DOI: 10.1111/jop.12264.

Ikeda N, Handa Y, Khim SP, Durward C, Axell T, Mizuno T, Fukano H, Kawai T. 1995.

Prevalence study of oral mucosal lesions in a selected Cambodian population. Community Dent Oral Epidemiol 23:49-54.

Kramer IR, Pindborg JJ, Bezroukov V, Infirri JS. 1980. Guide to epidemiology and diagnosis of oral mucosal diseases and conditions. World Health Organization. Community Dent Oral Epidemiol 8:1-26. DOI: 10.1111/j.1600-0528.1980.tb01249.x.

Kurago ZB. 2016. Etiology and pathogenesis of oral lichen planus: an overview. Oral Surg Oral Med Oral Pathol Oral Radiol 122:72-80. DOI: 10.1016/j.oooo.2016.03.011.

Mansour Ghanaei F, Joukar F, Rabiei M, Dadashzadeh A, Kord Valeshabad A. 2013. Prevalence of oral mucosal lesions in an adult Iranian population. Iran Red Crescent Med J 15:600604. DOI: $10.5812 /$ ircmj.4608.

Peer] reviewing PDF | (2019:08:40507:2:0:NEW 13 Jan 2020) 
323 McCullough MJ, Prasad G, Farah CS. 2010. Oral mucosal malignancy and potentially malignant 324 lesions: an update on the epidemiology, risk factors, diagnosis and management. Aust

325

326

327

328

329

330

331

332

333

334

335

336

337

338

339

340

341

342

343

344

345

346

347

348 Dent J 55 Suppl 1:61-65. DOI: 10.1111/j.1834-7819.2010.01200.x.

Mendes DC, Poswar Fde O, de Oliveira MV, Haikal DS, da Silveira MF, Martins AM, De Paula AM. 2012. Analysis of socio-demographic and systemic health factors and the normative conditions of oral health care in a population of the Brazilian elderly. Gerodontology 29:e206-214. DOI: 10.1111/j.1741-2358.2010.00446.x.

Mohamed S, Janakiram C. 2014. Recurrent aphthous ulcers among tobacco users- hospital based study. J Clin Diagn Res 8:ZC64-LC66. DOI: 10.7860/JCDR/2014/10368.5145.

Mumcu G, Cimilli H, Sur H, Hayran O, Atalay T. 2005. Prevalence and distribution of oral lesions: a cross-sectional study in Turkey. Oral Dis 11:81-87. DOI: 10.1111/j.16010825.2004.01062.x.

O'Sullivan EM. 2011. Prevalence of oral mucosal abnormalities in addiction treatment centre residents in Southern Ireland. Oral Oncol 47:395-399. DOI: 10.1016/j.oraloncology.2011.03.003.

Pentenero M, Broccoletti R, Carbone M, Conrotto D, Gandolfo S. 2008. The prevalence of oral mucosal lesions in adults from the Turin area. Oral Dis 14:356-366. DOI: 10.1111/j.1601-0825.2007.01391.x.

Petti S. 2003. Pooled estimate of world leukoplakia prevalence: a systematic review. Oral Oncol 39:770-780.

Porter SR, Mercadante V, Fedele S. 2017. Oral manifestations of systemic disease. Br Dent J 223:683-691. DOI: 10.1038/sj.bdj.2017.884.

Qi XQ. 2008. Report of Third National Survey of Oral Health Status in China. Beijing: People's Medical Publishing House.

Ribeiro AS, Salles PR, da Silva TA, Mesquita RA. 2010. A review of the nonsurgical treatment of oral leukoplakia. Int J Dent 2010:186018. DOI: 10.1155/2010/186018. 
349 Shulman JD, Beach MM, Rivera-Hidalgo F. 2004. The prevalence of oral mucosal lesions in

350

351

352

353

354

355

356

357

358

359

360

361

362

363

364

365

366

367

368

369

370

371

372

373

374

375
U.S. adults: data from the Third National Health and Nutrition Examination Survey, 1988-1994. J Am Dent Assoc 135:1279-1286.

Splieth CH, Sumnig W, Bessel F, John U, Kocher T. 2007. Prevalence of oral mucosal lesions in a representative population. Quintessence Int 38:23-29.

van der Meij EH, Schepman KP, van der Waal I. 2003. The possible premalignant character of oral lichen planus and oral lichenoid lesions: a prospective study. Oral Surg Oral Med Oral Pathol Oral Radiol Endod 96:164-171. DOI: 10.1016/S1079210403003056.

van Monsjou HS, Wreesmann VB, van den Brekel MW, Balm AJ. 2013. Head and neck squamous cell carcinoma in young patients. Oral Oncol 49:1097-1102. DOI: 10.1016/j.oraloncology.2013.09.001.

Villa A, Villa C, Abati S. 2011. Oral cancer and oral erythroplakia: an update and implication for clinicians. Aust Dent J 56:253-256. DOI: 10.1111/j.1834-7819.2011.01337.x.

Wang YY, Tail YH, Wang WC, Chen CY, Kao YH, Chen YK, Chen CH. 2014. Malignant transformation in 5071 southern Taiwanese patients with potentially malignant oral mucosal disorders. BMC Oral Health 14:99. DOI: 10.1186/1472-6831-14-99.

Warnakulasuriya S, Kovacevic T, Madden P, Coupland VH, Sperandio M, Odell E, Moller H. 2011. Factors predicting malignant transformation in oral potentially malignant disorders among patients accrued over a 10-year period in South East England. J Oral Pathol Med 40:677-683. DOI: 10.1111/j.1600-0714.2011.01054.x.

World Health Organization. 2013. Oral health surveys: basic methods. Fifth edition. Niigata: Niigata University.

Xu GQ, Shi HB, Qiu XY, Tang H. 1981. A report on oral mucosal diseases in 18,769 Shanghai residents. Stomatology 1:26-29. 


\section{Figure Legends}

378 Figure 1. Prevalence distribution of different types of OMDs among residents of different

379 genders.

380 Figure 2. Prevalence of different types of OMDs among residents of different age groups.

381 Figure 3. Risk factors of oral mucosal disease in community residents - smoking and drinking 382 habits.

383 Figure 4. Influence of mental state on OMDs of community residents.

384 Table 1. Analysis of risk factors for OMDs

385 Table 2. Analysis of risk factors for OLP

386 


\section{Table $\mathbf{1}$ (on next page)}

\section{Table 1. Analysis of risk factors for OMDs}

* univariate logistic regression

** multivariate logistic regression 
1 Table 1. Analysis of risk factors for OMDs

\begin{tabular}{|c|c|c|c|c|c|c|c|}
\hline$\square$ & variable & case & control & $\begin{array}{l}\text { Unadjusted OR* } \\
(\mathrm{OR} 95 \% \mathrm{CI})\end{array}$ & $\begin{array}{l}\mathrm{p} \\
\text { value* }\end{array}$ & $\begin{array}{l}\text { Unadjusted OR** } \\
(\mathrm{OR} 95 \% \mathrm{CI})\end{array}$ & $\begin{array}{l}\mathrm{p} \\
\text { value }^{* *}\end{array}$ \\
\hline \multirow[t]{3}{*}{ age } & $\geq 60$ & $34(6.4)$ & 495(93.6) & $0.893(0.113-7.03)$ & 0.914 & & \\
\hline & $40-59$ & $5(7.4)$ & $63(92.6)$ & $1.032(0.111-9.581)$ & 0,978 & & \\
\hline & $<40$ & $1(7.1)$ & 13(92.9) & 1 & & & \\
\hline \multirow[t]{3}{*}{ smoking } & yes & $5(7.0)$ & $66(93.0)$ & $1.107(0.417-2.934)$ & 0.839 & & \\
\hline & ex & $2(8.0)$ & $23(92.0)$ & $1.27(0.287-5.62)$ & 0.753 & & \\
\hline & no & $33(6.40)$ & 482(93.6) & 1 & & & \\
\hline \multirow[t]{3}{*}{ drink } & yes & $3(4.7)$ & $61(95.3)$ & $0.666(0.199-2.225)$ & 0.509 & & \\
\hline & ex & $0(0.0)$ & $9(100.0)$ & $0(0)$ & 0.999 & & \\
\hline & no & $37(6.9)$ & $501(93.1)$ & 1 & & & \\
\hline \multirow[t]{2}{*}{ hypertension } & yes & $14(4.7)$ & $287(95.3)$ & $0.533(0.273-1.041)$ & 0.066 & $0.45(0.224-0.906)$ & 0.025 \\
\hline & no & $26(8.4)$ & 284(91.6) & 1 & & & \\
\hline diabetes & yes & $10(10.5)$ & $85(89.5)$ & $1.906(0.899-4.042)$ & 0.093 & $2.41(1.098-5.294)$ & 0.028 \\
\hline mellitus & no & $30(5.8)$ & $486(94.2)$ & 1 & & & \\
\hline coronary heart & yes & $4(5.2)$ & $73(94.8)$ & $0.758(0.262-2.192)$ & 0.609 & & \\
\hline disease & no & $36(6.7)$ & 498(93.3) & 1 & & & \\
\hline cerebrovascular & yes & $3(8.8)$ & $31(91.2)$ & $1.412(0.412-4.837)$ & 0.582 & & \\
\hline disease & no & $37(6.4)$ & $540(93.6)$ & 1 & & & \\
\hline
\end{tabular}

$2 *$ univariate logistic regression

$3 * *$ multivariate logistic regression 


\section{Table 2 (on next page)}

\section{Table 2. Analysis of risk factors for OLP}

* univariate logistic regression

** multivariate logistic regression 
1 Table 2. Analysis of risk factors for OLP

\begin{tabular}{|c|c|c|c|c|c|c|c|}
\hline$\square$ & variable & case & control & $\begin{array}{l}\text { Unadjusted OR* } \\
\text { (OR 95\%CI) }\end{array}$ & $\begin{array}{l}\mathrm{p} \\
\text { value* }\end{array}$ & $\begin{array}{l}\text { Unadjusted OR** } \\
\text { (OR } 95 \% \mathrm{CI})\end{array}$ & $\begin{array}{l}\mathrm{p} \\
\text { value** }\end{array}$ \\
\hline \multirow[t]{3}{*}{ age } & $\geq 60$ & $5(0.9)$ & $524(99.1)$ & $0.124(0.014-1.138)$ & 0.065 & $0.030(0.002-0.396)$ & 0.008 \\
\hline & $40-59$ & $3(4.4)$ & $65(95.6)$ & $0.6(0.058-6.230)$ & 0.669 & $0.286(0.022-3.717)$ & 0.339 \\
\hline & $<40$ & $1(7.1)$ & 13(92.9) & 1 & & & \\
\hline \multirow[t]{3}{*}{ smoking } & yes & $3(4.2)$ & $68(95.8)$ & $4.5(1.052-19.252)$ & 0.043 & $8.732(1.773-43.013)$ & 0.008 \\
\hline & ex & 1(4) & $24(96)$ & $4.25(0.478-37.812)$ & 0.194 & $4.497(0.368-55.002)$ & 0.239 \\
\hline & no & $5(1)$ & $510(99)$ & 1 & & & \\
\hline \multirow[t]{3}{*}{ drink } & yes & $2(3.1)$ & $62(96.9)$ & $2.447(0.497-12.04)$ & 0.271 & & \\
\hline & ex & $0(0)$ & $9(100)$ & $0(0)$ & 0 & & \\
\hline & no & $7(1.3)$ & $531(98.7)$ & 1 & & & \\
\hline \multirow[t]{2}{*}{ hypertension } & yes & $6(1.9)$ & $304(98.1)$ & $0.51(0.126-2.058)$ & 0.344 & & \\
\hline & no & $3(1)$ & 298(99) & 1 & & & \\
\hline diabetes & yes & $5(5.3)$ & $90(94.7)$ & 7.111(1.874-26.988) & 0.004 & $14.083(2.958-67.05)$ & 0.001 \\
\hline mellitus & no & $4(0.8)$ & $512(99.2)$ & 1 & & 1 & \\
\hline coronary heart & yes & $1(1.3)$ & $76(98.7)$ & $0.865(0.107-7.014)$ & 0.892 & & \\
\hline disease & no & $8(1.5)$ & $526(98.5)$ & 1 & & & \\
\hline cerebrovascular & yes & $1(2.9)$ & $33(97.1)$ & $2.155(0.262-17.747)$ & 0.475 & & \\
\hline disease & no & $8(1.4)$ & $526(98.6)$ & 1 & & & \\
\hline
\end{tabular}

$2 *$ univariate logistic regression

$3 * *$ multivariate logistic regression 
Figure 1

Figure 1. Prevalence distribution of different types of OMDs among residents of different genders.

Distribution condition of various diseases in male and female

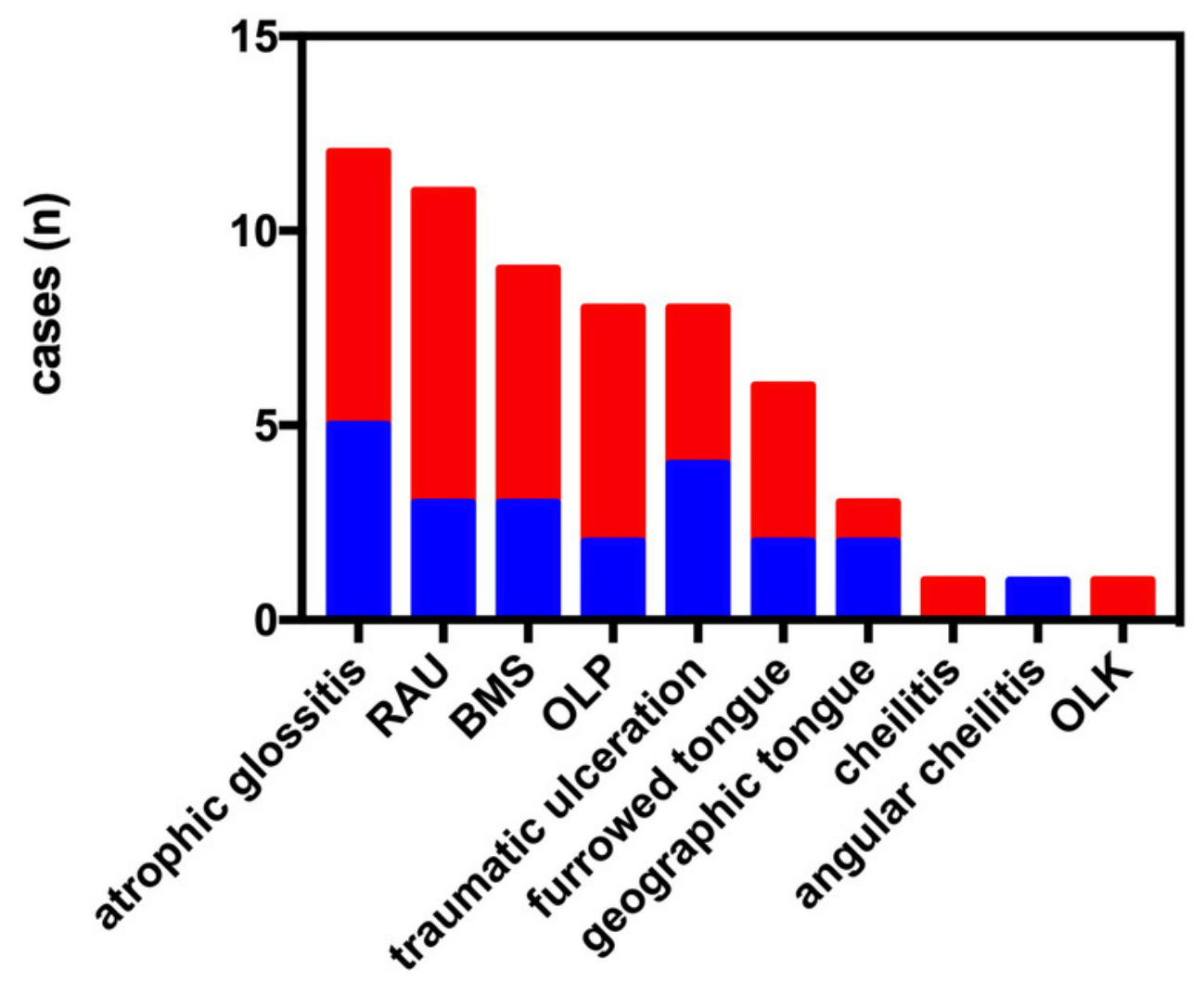

Female

Male 
Figure 2

Figure 2. Prevalence of different types of OMDs in residents of different age groups (disease prevalence trend of residents of different ages.

\section{Distribution condition of various diseases in various ages of resident}
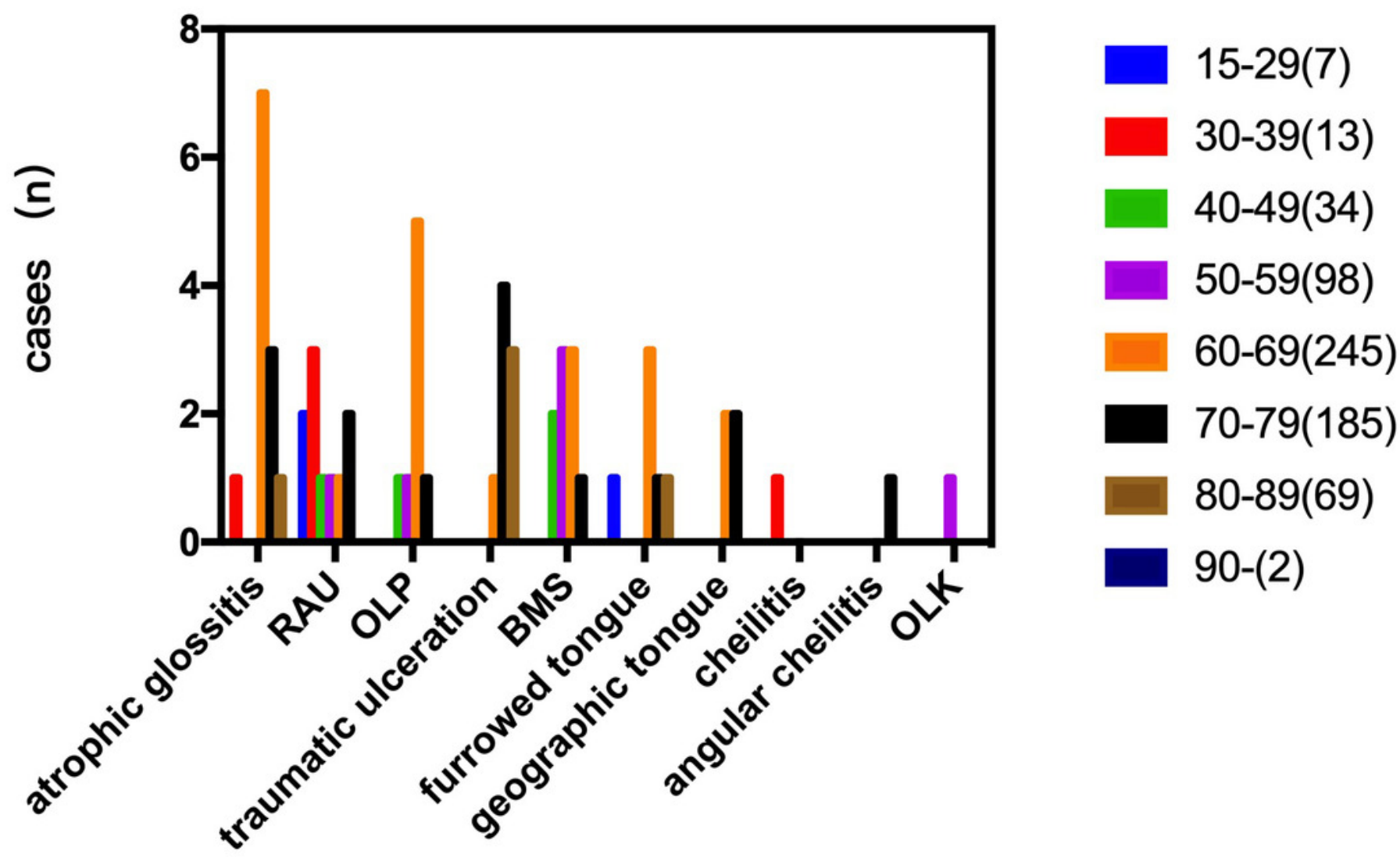
Figure 3

Figure 3. Risk factors of oral mucosal disease in community residents - smoking and drinking habits.

(a) Effect of smoking for OMDs

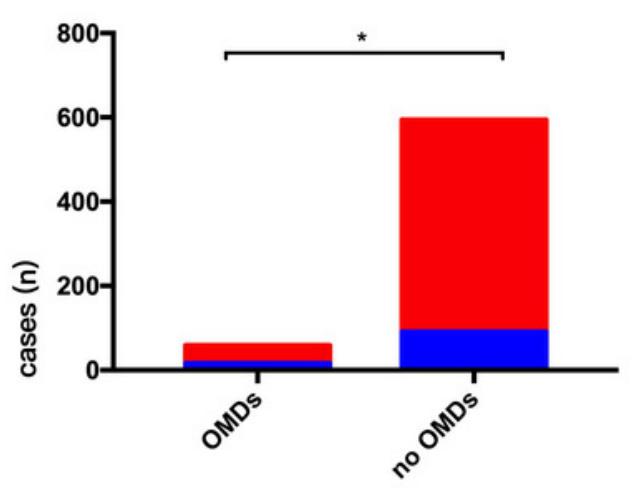

(b) Effect of drink for OMDs

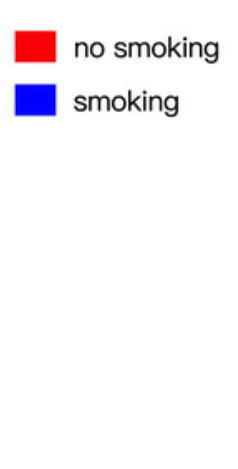

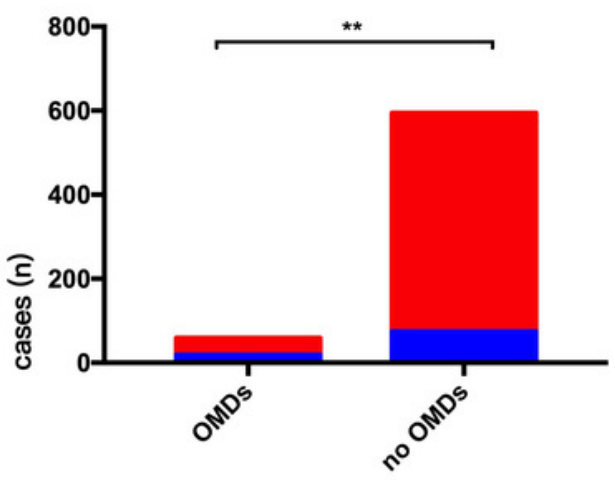

no drink

drink 
Figure 4

Figure 4. Influence of mental state on OMDs of community residents.

(a) Effect of anxiety for OMDs

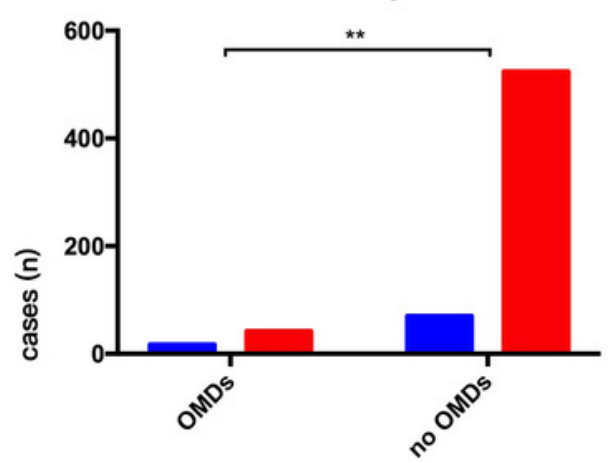

(b) Effect of stress for OMDs
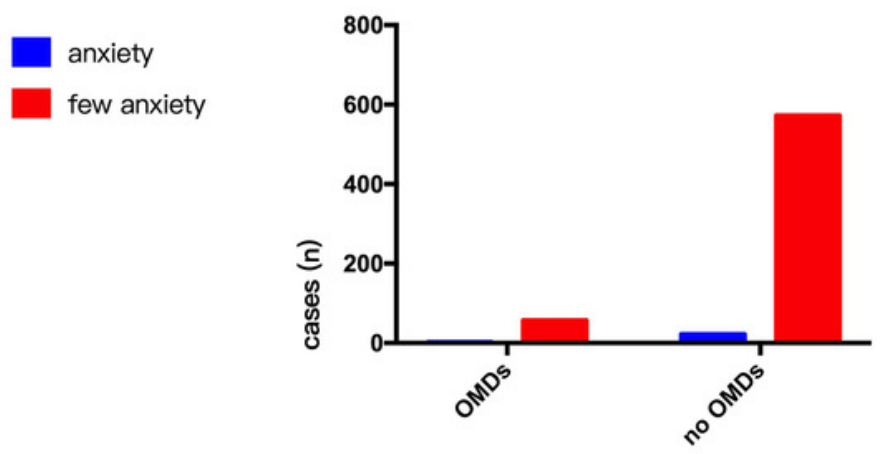

stress

few stress 